Abstract P043 Table 1 Baseline characteristics; data are mean $\left(25^{\text {th }}, 75^{\text {th }}\right.$ percentiles $)$

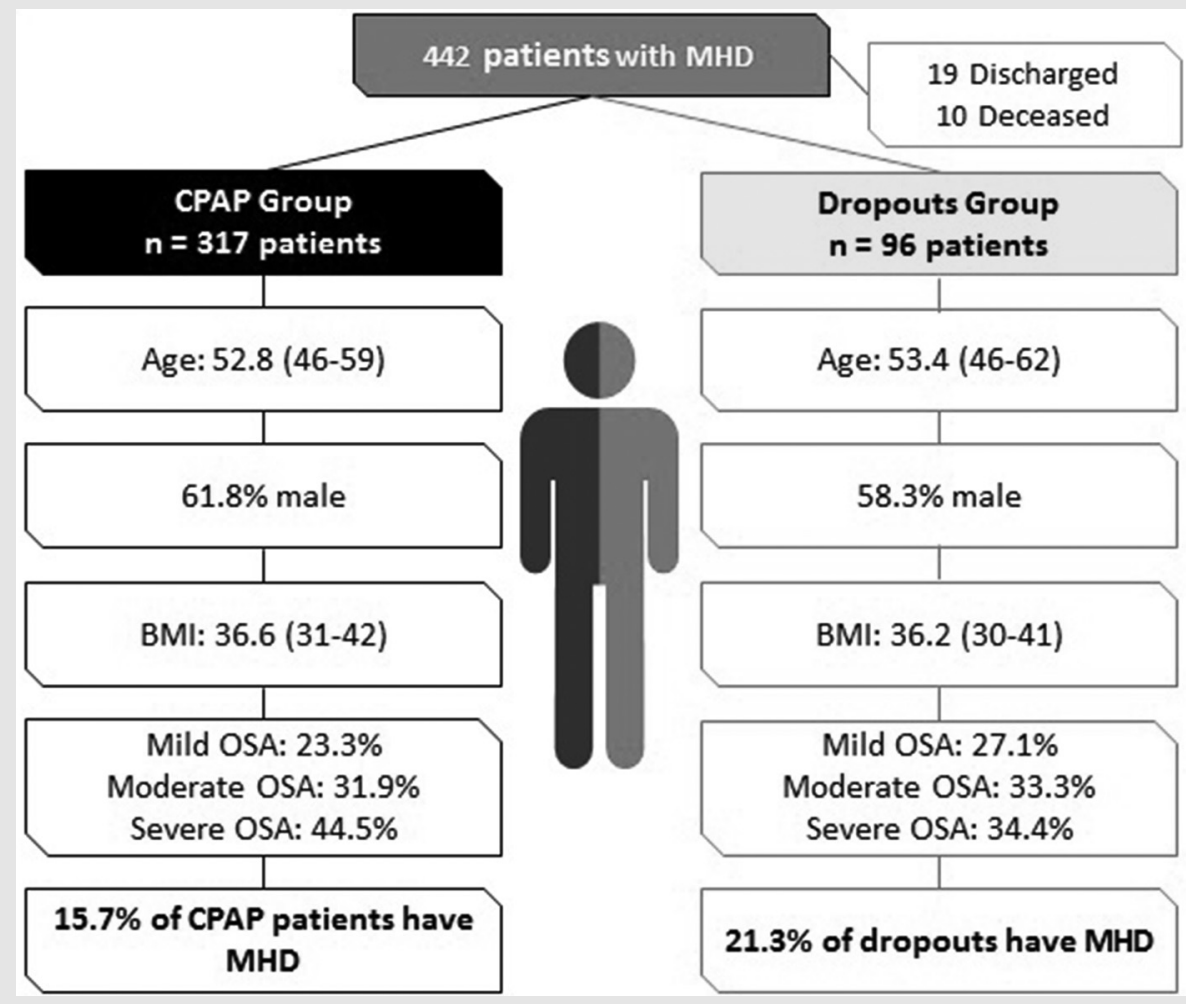

Within the MHD group, there was similar age, gender distribution and BMI. [Table 1].

Discussion The CPAP treatment adherence rate in our service is $76.4 \%$, which is higher than reported in literature. The percentage of patients with MHD is lower than expected $(16.7 \%)$, as it is estimated that $39 \%$ of the adults in the UK suffer with common mental health disorders. There is a higher percentage of $\mathrm{MHD}$ in the dropout group $(21.29 \%)$ than in the CPAP group (15.71\%), despite similar age, BMI and gender distribution, suggesting this may be a factor in achieving compliance with CPAP treatment.

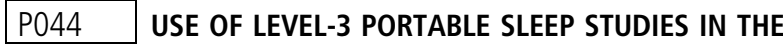 PAEDIATRIC POPULATION. USER FEEDBACK}

Sakina Dastagir*, Rishi Pabary, Ross Langley, Ridma Jayarathna, Hannah Williams. Royal Brompton, London, UK

\subsection{6/bmiresp-2019-bssconf.44}

Introduction Ambulatory sleep, cardio-respiratory devices, or level 3 sleep studies have been used for screening and diagnosis of sleep apnoea in the adult and paediatric population. The advantages of using a level 3 portable sleep monitoring device include being able to expedite diagnosis, ease for the patient and family members and being 50\% the cost of an in-patient level 1 PSG. Literature is limited on the use of these devices in paediatrics, this includes patient/guardian usability and satisfaction as well as the ability to assess sleep disorders.

From May 2018 we produced and distributed a questionnaire to address these issues.
Methods The questionnaire comprised of 4 questions about the device and the sleep study process. There are 3-6 options for each answer (see table 1). We also recorded technical failures using the report generated by the DOMINO LightTM Software auto-analyser. This includes failure rate of the pulse oximeter, respiratory bands and nasal cannulae.

Results At the time of analysis, 30 completed questionnaires demonstrated that the majority (93\%) would prefer a home study compared to an in-patient study. At the time of analysis, 31 completed questionnaire revealed that $48 \%$ of guardians found the most challenging part of the home set up was the nasal cannulae. Figure 1.

Discussion The preference for home studies suggests this method of monitoring and diagnosing patients could be beneficial for the future of our service. This is in keeping with the desire to deliver modern healthcare within the home setting so called 'hospital at home'.

The nasal cannulae provides important information for analysis and diagnosis of the study. For further analysis, the

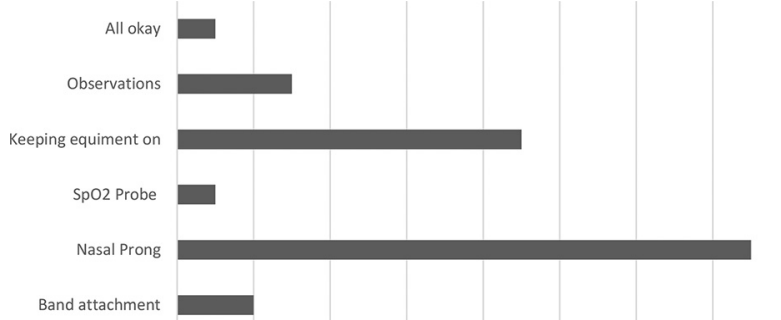

Abstract P044 Figure 1 Graph showing guardian response to the most challenging Equipment to set up 
Abstract P044 Table 1 Scores for each question

\begin{tabular}{|c|c|c|c|}
\hline \multicolumn{4}{|c|}{ Question and corresponding answer } \\
\hline Q1: Ease of set up & $\begin{array}{c}\text { Q2: Most challenging } \\
\text { during study }\end{array}$ & $\begin{array}{c}\text { Q3: Most challenging } \\
\text { during process }\end{array}$ & Home/Ward \\
\hline Very Easy & Band attachment & Collecting equipment & Home \\
\hline Easy & Nasal Prong & Learning how to use & Ward \\
\hline Ok & SpO2 Probe & Return of equipment & \\
\hline Difficult & Keeping equpiment on & & \\
\hline Very Difficult & Observations & & \\
\hline & All Ok & & \\
\hline
\end{tabular}

frequent technical failures which could occur in the sensors from Question 2 (table 1.) could be analysed.

\section{P045 AGGREGATED PATIENT JOURNEYS AND NO-SHOW RATES OF OXIMETRY OUTREACH NETWORK IN EAST ANGLIA}

${ }^{1}$ Kieran Lee*, ${ }^{2}$ Natalia Andrienko, ${ }^{2}$ Gennady Andrienko, ${ }^{3}$ Ibad Kureshi, ${ }^{4}$ Toni Staykova, ${ }^{1}$ Ian Smith. ${ }^{1}$ Royal Papworth Hospital, Cambridge, UK; ${ }^{2}$ Fraunhofer Institute, Sankt Augustin, Germany; ${ }^{3}$ Inlecom Systems, Brussels, Belgium; ${ }^{4}$ Cambridge Medical Academy, Cambridge, UK

\subsection{6/bmjresp-2019-bssconf.45}

Introduction Royal Papworth Hospital performs >10,000 home oximetry tests p.a. across a large area with challenging road-infrastructure. We have developed a distributed system of oximetry exchange facilities (EF's) at outreach-clinics and GP surgeries over several years without a 'masterplan'. As part of the Track and Know project (EU2020) we examined relationships between the distribution of patients and EF's and noshows.

Method Data for 5 years' (2013-2018) of oximetry tests with patient addresses and clinic outcomes were used to reconstruct likely journeys applying a bespoke tessellation algorithm, dividing the territory into polygons enclosing clusters of patients" homes and at most one EF. For each pair of compartments (P\&Q), we computed the number of appointments for patients in $\mathrm{P}$ to the clinic in $\mathrm{Q}$ and the $\%$ of no-shows. These were mapped (see figure 1). Data were collected for patient demographics and the travel information was enriched with weather conditions on the appointment day.

Results Data from 46,211 planned pick up's were examined. There was a high no-show rate $(15.8 \%)$ and an uneven distribution of journeys to each pick-up site. Despite the network containing 21 sites $>50 \%$ of pick-ups were scheduled for the hospital. No show rates were not related to travel distance and were more likely in good weather than bad. We have recalculated that the service could be better provided from 10 EF's.

Discussion We have re-evaluated our service using complex patient flow metrics and the results have confounded some predictions. Distance alone does not predict no-show rate and we plan to explore the impact of public transport and more sophisticated patient based factors. We plan to redesign the EF distribution network in line with our outcomes with the goal of maximising efficiency and will revisit whether we can improve accessibility and reduce our no-show rate while reducing clinic overheads.

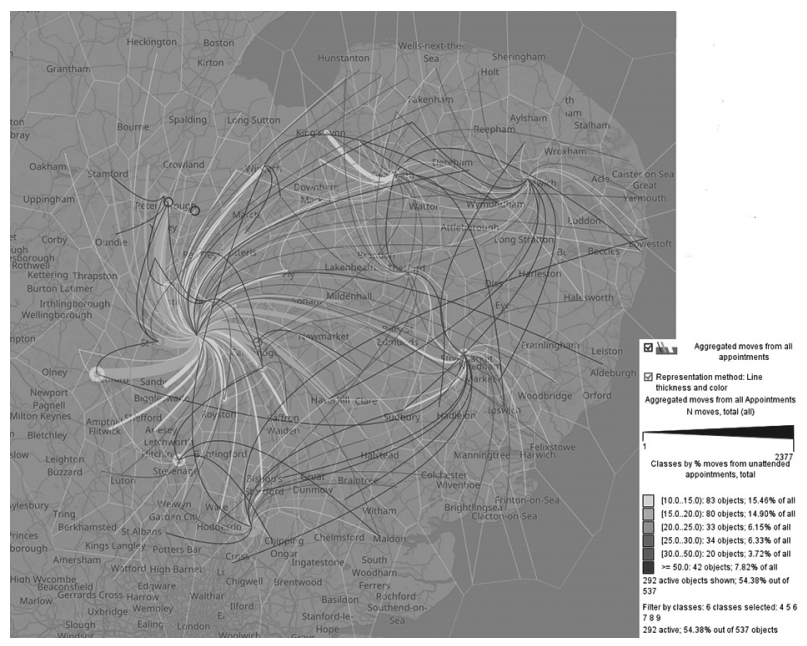

Abstract P045 Figure 1 